\title{
Receding contact lines: from sliding drops to immersion lithography
}

\author{
K.G. Winkels ${ }^{1, a}$, I. R. Peters ${ }^{1}$, F. Evangelista ${ }^{2}$, M. Riepen ${ }^{2}$, A.Daerr ${ }^{3}$, L.Limat ${ }^{3}$, and J.H. \\ Snoeijer ${ }^{1}$ \\ 1 Physics of Fluids Group, University of Twente, The Netherlands \\ 2 ASML BV, Veldhoven, The Netherlands \\ ${ }^{3}$ Laboratoire MSC, Universite Paris Diderot, France
}

\begin{abstract}
Instabilities of receding contact lines often occur through the formation of a corner with a very sharp tip. These dewetting structures also appear in the technology of Immersion Lithography, where water is put between the lens and the silicon wafer to increase the optical resolution. In this paper we aim to compare corners appearing in Immersion Lithography to those at the tail of gravity driven-drops sliding down an incline. We use high speed recordings to measure the dynamic contact angle and the sharpness of the corner, for varying contact line velocity. It is found that these quantities behave very similarly for Immersion Lithography and drops on an incline. In addition, the results agree well with predictions by a lubrication model for cornered contact lines, hinting at a generic structure of dewetting corners.
\end{abstract}

\section{Introduction}

A fluid displacing another immiscible fluid across a solid surface is a very common phenomenon in both nature (e.g. such water drops sliding down a leaf [1]) and industrial processes (e.g. printing and coating [2]). The fluid motion is controlled by the dynamics of the contact line, which is susceptible to instabilities when moving at large speeds $[1,3]$. The standard approach to this problem is to assume the contact line remains straight during the motion, so that the flow is essentially two-dimensional. At low speeds this gives rise to the Cox-Voinov law [4,5], describing the dynamic contact angle as a function of speed. The relevant dimensionless parameter is the capillary number, $\mathrm{Ca}=U \eta / \gamma$, representing the contact line velocity $U$ rescaled by viscosity $\eta$ and surface tension $\gamma$ respectively. The simplest scenario for instability of straight contact lines is that the contact angle becomes zero at a critical speed [6,7]. At higher values of Ca the contact line gives way to the deposition of a liquid film $[6,8-11]$.

Interestingly, instability of receding contact lines often occurs through the formation of a corner with a very sharp tip, for which the flow is manifestly three-dimensional. This goes back to the work of Blake and Ruschak [12], who found that in the withdrawal of a plate from a liquid bath, the contact line adopts a 'v' shape at large speeds. The appearance of the corner was interpreted as a mechanism to reduce the effective contact line speed, since the inclination of the contact lines reduces their normal (perpendicular) velocity. Very similar corner shapes were observed for drops sliding down an incline, as shown in figure 1b [13,14]. Drops are viewed from the top while sliding down under the influence of gravity. Instead of remaining circular, the receding 'tail' of the drop develops a corner-like structure that turns sharper with increasing capillary number. At even higher speeds the tail breaks up and small droplets are deposited on

\footnotetext{
a e-mail: k.g.winkels@utwente.nl
} 
the surface.Under the assumption of a conical structure of the liquid, this tail can be described by a lubrication model [15]. This captures the essential geometrical features as well as the flow in the vicinity of the contact line $[16,17]$.

These three-dimensional dewetting phenomena turn out to play a crucial role in the technology of Immersion Lithography (figure 1c and 1d). This is a technique that is widely applied in semiconductor industry to achieve higher optical resolutions, and accordingly smaller dimensions $[18,19]$. The conventional Lithography method, which consists of the projection of light patterns through a lens on substrates, is improved by replacing the air between the lens and the substrate by a liquid with a higher refractive index (figure 1c). This increases the numerical aperture of the system and consequently the imaging resolution. For ultra pure water, the commonly used liquid, the smallest printable features become of order $\sim 40 \mathrm{~nm}$. While the liquid is advantageous for the optical performances, it introduces new complexities associated to the contact line. Since the lens cannot cover the complete substrate at once, it is moved over the wafer with speeds close to instability: the critical velocity at which water loss occurs near the contact line determines the maximum speed of the lens. As such, it provides a tremendous limitation for the production yield of Immersion Lithography.
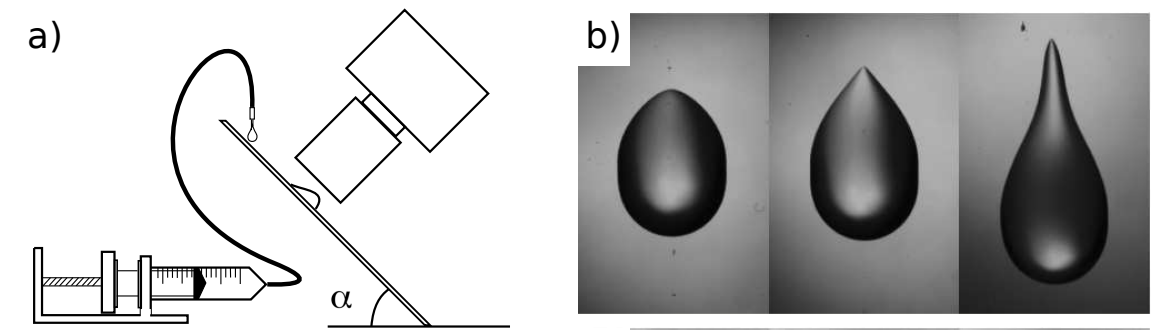

c)
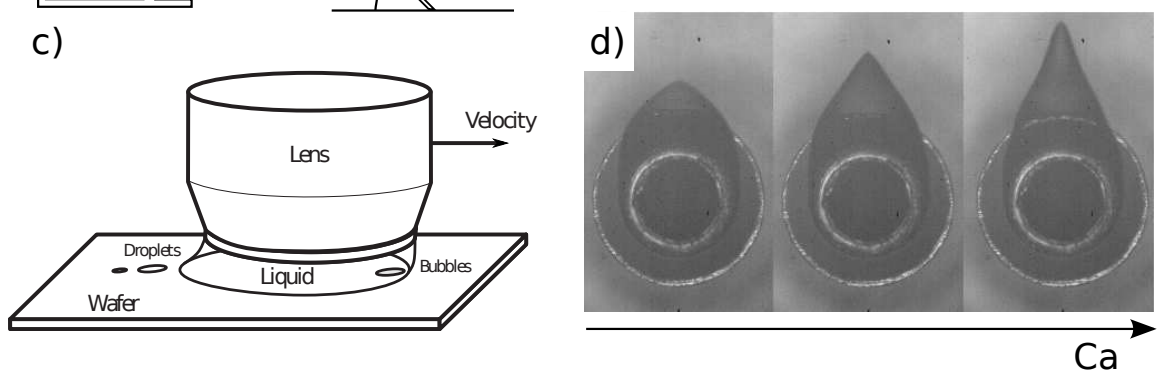

Fig. 1: Analogy between drops sliding down an inclined plate and moving contact lines in Immersion Lithography. a) Setup used to study droplets sliding down an inclined plane; b) images from the experimental setup as shown in (a); c) Sketch of an Immersion Lithography system; d) images from a setup mimicking an Immersion Lithography system.

In this paper we experimentally investigate the v-shaped contact lines observed in Immersion Lithography. We follow the evolution of the liquid corner as a function of speed by monitoring the angles from side-view and top-view imaging. It is clear from figure 1 that there is a striking resemblance with drops sliding down a plane and we make a quantitative comparison between the two systems. There are, however, some important differences between the two systems. First, the drops on an incline are driven by gravity and the speed is adjusted by the angle of inclination $\alpha$, while in the Immersion Lithography experiment the substrate speed is imposed and controlled by a motor. Second, the Reynolds number for the silicon oil drops is negligible, while inertial effects could be expected for the low viscosity water drops in Immersion Lithography. These differences do not affect the generic features of the cornered contact lines in the set of experimental parameters/setting used, although some quantitative differences are indeed observed. 
The paper is organized as follows. Section 2 defines the key quantities of the corner geometry and briefly reviews the main theoretical results. A detailed description of this experimental setup is given in Sec. 3. The central results are presented in Sec. 4, where we also make the detailed comparison with sliding drop data from [20], and the paper closes with a Discussion.

\section{Three-dimensional dewetting: cone angles}

The standard description of wetting dynamics assumes a straight contact line, resulting in a flow that is effectively two-dimensional. If one further assumes that inertia is not important, the dynamic contact angle seen from a side view, $\theta$, follows from a balance of viscous forces and surface tension and thus depends on the capillary number Ca. Away from the critical speed, this is accurately described by the Cox-Voinov relation $[4,5]$

$$
\theta^{3}=\theta_{0}^{3}-9 \mathrm{Ca} \ln \left(\frac{x}{\ell}\right)
$$

where $\theta_{0}$ is the static contact angle, while $\ell$ is a microscopic length at which the viscous singularity is regularized [21]. Note that the value of $\theta$ depends logarithmically on $x$, the distance to the contact line. Experimentally, the observed slope thus depends on the length scale at which the measurement is performed [22-24].

In the situation of straight contact lines the only interface curvature is directed along the motion of the contact line. This approach is too simple, however, as for the drops shown in figure 1 the interface becomes truly three-dimensional at higher Ca. The three-dimensional geometry can be solved by assuming that the interface develops a sharp conical shape [15-17]. The cone is characterised by two angles (figure 2): side view angle $\theta$ and top view (opening) angle $\phi$. The lubrication equations indeed admit similarity solutions that are consistent with such a conical structure. This lubrication approach gives explicit predictions for the cone angles and these will be measured for the drops in figure 1.

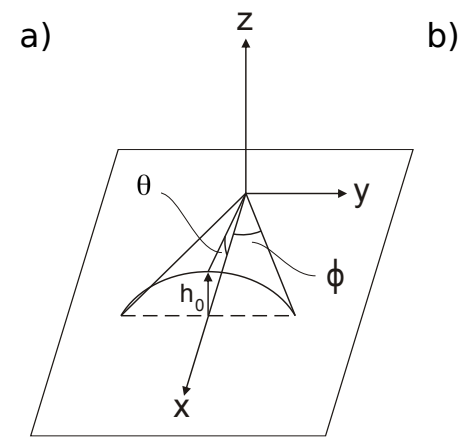

b)

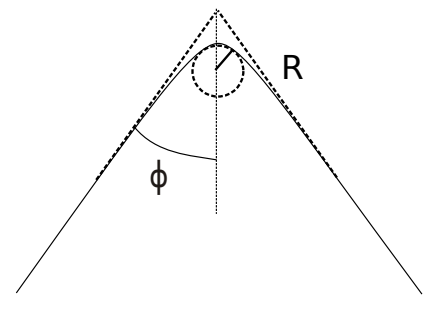

Fig. 2: Schematic view of a cornered receding contact line. a) three-dimensional cone structure with characteristic angles $\phi$ and $\theta, \mathrm{b}$ ) the tip of the corner has a finite radius of curvature $\mathrm{R}$.

A first prediction is that the cone angles $\theta$ and $\phi$ are related according to $[15,16]$

$$
\tan ^{3} \theta=\frac{35}{16} \mathrm{Catan}^{2} \phi .
$$

This expresses the balance between viscous dissipation and capillary forces. In contrast to (1) the side-view angle no longer depends on the distance to the contact line, but $\theta$ is simply constant along the cone. The reason is that for the cone the capillary forces result from the interface curvature perpendicular to the direction of motion (for straight contact lines this perpendicular curvature is zero). One should note, however, that this is not yet a closed expression for $\theta$ as 
a function of speed, since the opening angle $\phi$ also depends on Ca. The problem can be closed by introducing a matching condition near the inclined contact lines, and yields [17]

$$
\frac{C a}{\theta_{0}^{3}}=\frac{2 \sin \phi}{18 \sin ^{2} \phi \ln \left(\frac{L}{\ell}\right)+\frac{35}{\cos ^{2} \phi}} \approx \frac{2 \phi}{18 \phi^{2} \ln \left(\frac{L}{\ell}\right)+35} .
$$

The latter step represents the limit of small $\phi$. This relation predicts a maximum speed for the cone, occurring at a minimum value of the opening angle $\phi$. Higher speeds result into instabilities and break-up.

While the cone model assumes a tip that is infinitely sharp, the tip of the receding contact line is in reality rounded at a small scale (figure 1). We therefore define the radius of curvature of the tip $R$ as shown in figure $2 \mathrm{~b}$. At a large distance from the tip, $x \gg R$, one recovers the conical geometry characterized by a constant angle $\theta$. On the other hand, the contact line will appear nearly straight when approaching the tip at a small distance $x \ll R$. We thus expect to recover the Cox-Voinov relation (1) in this regime. As was shown in [20], these two regions should match on a length scale of order $R$. Since in the corner regime $\theta^{3} \ll \theta_{0}^{3}$, this matching yields

$$
0 \approx \theta_{0}^{3}-9 \mathrm{Ca} \ln \left(\frac{R}{\ell}\right)
$$

where we took $x \approx R$ and $\theta \approx 0$ in (1). By inverting (4), we find a prediction for the tip curvature $R$ in terms of the speed:

$$
R \approx \ell \exp \left(\frac{\theta_{0}^{3}}{9 \mathrm{Ca}}\right)
$$

In the remainder of the paper we will experimentally measure the angles $\theta$ and $\phi$ as well as the tip curvature $R$, for varying contact line velocities. The relations mentioned above will provide the framework to quantitatively compare the data taken from the Immersion Lithography setup and from the drops sliding down an incline.

\section{Experimental setup}

The data compared in this paper comes from two types of experiments: drops sliding down an inclined plate and moving contact lines in a configuration mimicking an Immersion Lithography system (figure 1). The data for the sliding drops on the inclined plane are taken from Peters et al. [20] and LeGrand et al. [14], in which drops of silicon oil were used with viscosities ranging from 10 to $1035 \mathrm{cP}$. The substrate was made partially wetting by a fluoro-polymers coating (FC725 by 3M). The various publications report minor differences in wetting properties, but typical values for the static contact angles are $\theta_{0} \sim 45^{\circ}$, with a hysteresis of $\sim 10^{\circ}$. Drops were created in a controlled manner by using a syringe pump, resulting in droplet volumes in the range of $6-10 \mathrm{~mm}^{3}$. The sliding speed of the drop was varied by the inclination angle of the plate.

The Immersion Lithography setup is shown in figure 3. While the sliding drops move through the reference frame of the camera, the liquid in Immersion Lithography is fixed in the reference frame of the cameras. The basic idea is to rotate a glass wafer on a turntable, while keeping a drop at a fixed position. To achieve this a droplet is held by the needle system shown in figure 3a. It consists of two concentric needles of different diameter (outer diameters of 1.84 $\mathrm{mm}$ and $1.27 \mathrm{~mm}$, for the outer and inner needle respectively). Standard pure water is supplied through the inner needle at a constant flow rate of $12 \mathrm{ml} / \mathrm{min}$. The outer needle simultaneously extracts water and air at a fixed rate of $2.4 \mathrm{~L} / \mathrm{min}$, so that the water is constantly refreshed. The wall thickness of the outer needle is kept very thin and the height of the needle above the substrate as heigh as possible $(250 \mu \mathrm{m})$. While holding the droplet, a coated glass wafer of $300 \mathrm{~mm}$ diameter and $0.7 \mathrm{~mm}$ thickness is moved underneath. This is done by rotating the wafer by a custom made motor (IBS Precision Engineering) that is able to rotate the wafer at 
a)

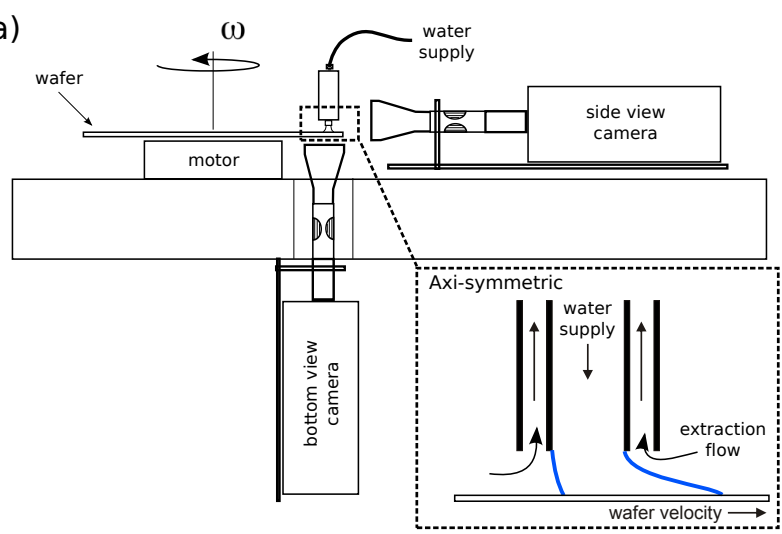

b)

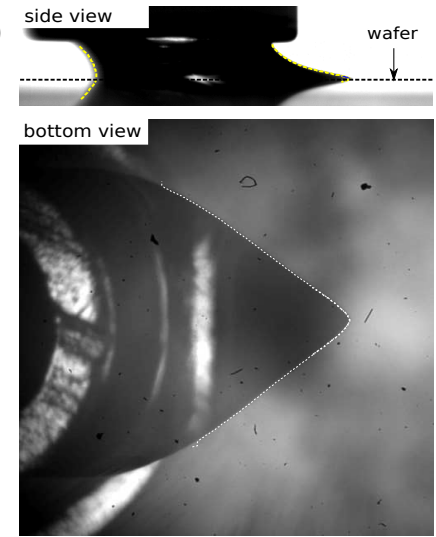

Fig. 3: a) Sketch of the Immersion Lithography setup b) Top and bottom view images from a typical measurement $(\mathrm{U}=604 \mathrm{~mm} / \mathrm{s})$. The dashed line represents the detected boundary of the droplet obtained from digital imaging analysis.

a maximum linear velocity of $u_{\max }=3 \mathrm{~m} / \mathrm{s}$ and maximum linear acceleration of $a_{\max }=100$ $\mathrm{m} / \mathrm{s}^{2}$ (measured at a radial distance of $140 \mathrm{~mm}$ from the center of the wafer). To obtain a partial wetting substrate, the glass wafers are coated having a static receding and advancing contact angle of $65^{\circ}$ and $87^{\circ}$, respectively.

We simultaneously image the drop from the side and from below (figure 3 ). For side view imaging a high speed camera (Photron SA 1.1) is used in combination with a long distance microscope (Navitar 12X Telecentric Zoom System), whereas for the bottom view a more detailed view is realised (Photron SA 1.1 in combination with a Navitar $12 \mathrm{X}$ long distance microscope attached to a Mitutoyo Infinity Corrected objective). Resolutions of $6 \mu \mathrm{m} /$ pixel (side) and 2.7 $\mu \mathrm{m} /$ pixel (bottom) are achieved. Typical recordings obtained from this setup are shown in figure 3b. Digital imaging analysis is used to detect the droplet boundary position. In both, bottom and side view recordings, this position is defined as the position of the maximum gradient in pixel-intensity values (8-bit image) in the region near the droplet contact line after image background subtraction. To find the gradient a Canny filter is used [25]. To avoid false detections, the edge detection is checked and verified by superposition on the original image (similar to the dashed lines shown in figure $3 \mathrm{~b}$ ). This boundary detection method is limited to pixel resolution and therefore results in discrete steps in the detected droplet edge. In order to find the tip position (wafer position) on sub-pixel level, a parabolic function is fit to the first 10 pixels closest to one of the uttermost pixels of the raw edge detection. The minimum of the parabolic function is taken as the tip position. The final steps of data analysis will be specified while discussing the results in the following section.

Experimental data is obtained from multiple runs on different wafers in order to check the reproducibility. During each run we increase the velocity to $1 \mathrm{~m} / \mathrm{s}$, with an acceleration of 1 $\mathrm{m} / \mathrm{s}^{2}$, while the droplet is recorded simultaneously from the side and bottom, at a frame rate of $1000 \mathrm{fps}$. This corresponds to a linear velocity increase of $1 \mathrm{~mm} / \mathrm{s}$ per frame. Hence, from a single recording it is possible to characterize the moving contact line behaviour as a function of the wafer speed. We have verified that the results are reproducible over a range of accelerations, so we can use the instantaneous speed (capillary number) as the relevant parameter. It should be noted that the Reynolds number based on the flying height is of the order $\mathcal{O}(100)$. 


\section{Results}

\subsection{Dynamic contact angle}

The most basic characterisation of a moving contact line is the dynamic contact angle $\theta$. In the literature we find a number of different definitions and is still subject to discussion $[1,22$, 26]. Here we define $\theta$ as the local interface slope defined by a linear fit, at a fixed distance from the contact line. The measurement is based on the first detected boundary pixels within a fixed distance of $50 \mu \mathrm{m}$ from the tip measured along the interface. In the spirit of the CoxVoinov relation (1), this means that we measure the dynamic contact angle at a fixed position of $x=25 \mu \mathrm{m}$.
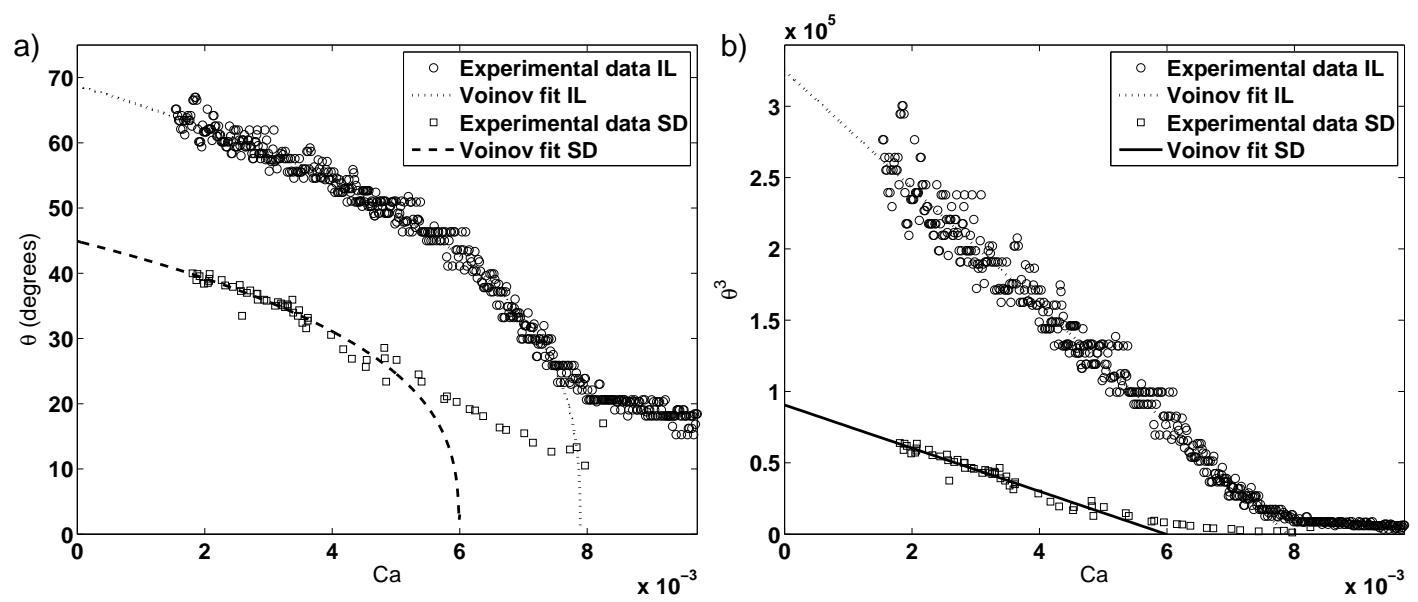

Fig. 4: Dynamic contact angle $\theta$ as a function of the capillary number (normalized contact line velocity). The circles and squares represent the data obtained from the Immersion Lithography setup (IL) and sliding droplet experiment (SD), respectively. The dashed lines represent the fit based on Cox-Voinov relation (1).

The results, up to break up, are presented in figure 4a. The circles represent data from Immersion Lithography, while the squares correspond to sliding drops. In both cases the dynamic contact angle decreases with $\mathrm{Ca}$ as expected for receding contact lines. For small speeds we find excellent agreements with the Cox-Voinov law, which is shown as dashed lines. The quality of the fits is further illustrated by plotting the same data as $\theta^{3}$ versus Ca (figure $4 \mathrm{~b}$ ). This gives a straight line for small speeds. According to (1), the slope of this line can be interpreted in terms of $\ln x / \ell$, where $x$ is the scale of the measurement $(\approx 25 \mu \mathrm{m})$ and $\ell$ a characteristic microscopic length. For the silicon oil drops this gives $\ln x / \ell=8.9$, which indeed corresponds to a microscopic length, $\ell \approx 7 \cdot 10^{-9} \mathrm{~m}$. The Immersion Lithography data give a value $\ln x / \ell=23$ $\left(\ell \approx 2.6 \cdot 10^{-15} \mathrm{~m}\right)$ which is surprisingly high (small). However, this value is consistent with experiments on water drops sliding down an incline [13], suggesting that it is specific for water.

Interestingly, a significant deviation from Cox-Voinov is observed at higher speeds for both data sets. For Immersion Lithography this occurs around $\mathrm{Ca} \sim 8 \cdot 10^{-3}$, while for sliding drops $\mathrm{Ca} \sim 6 \cdot 10^{-3}$. Below we show in detail that for both experiments this coincides with the moment that the scale of measurement $x$ becomes, $x \sim R$. We shall thus interpret this behaviour for $\theta$ as a change in geometry of the interface: from a two-dimensional straight contact line, as assumed for (1), to a three-dimensional conical shape. Note that the extrapolation of Voinov (two-dimensional approach) predicts vanishing contact angles. By changing the shape it thus seems that it can postpone the instabilities. However break up cannot be avoided, and occurs in the region of the maximum Ca plotted in the graph (for Immersion Lithography, $\mathrm{Ca} \sim 0.01$ and sliding droplets $C a \sim 0.008)$. 


\subsection{Tip curvature}

Let us now investigate the formation of the corner in more detail. The images on figure 5a show the change from a rounded contact line at low speeds to a sharp corner at higher speeds. To quantify this transition one can measure the curvature (sharpness) of the tip as a function of speed. We do so by fitting a circle to a number of detected boundary points near the tip. The fit is made in a least square sense, however the best fit will also depend on how many points are taken into account. To solve this, the best fit is always based on all points within a vertical distance of 5 pixels from the tip. A number which is based on the assumption that one cannot measure radii smaller than 5 pixels $(\sim 14 \mu \mathrm{m})$,

a)

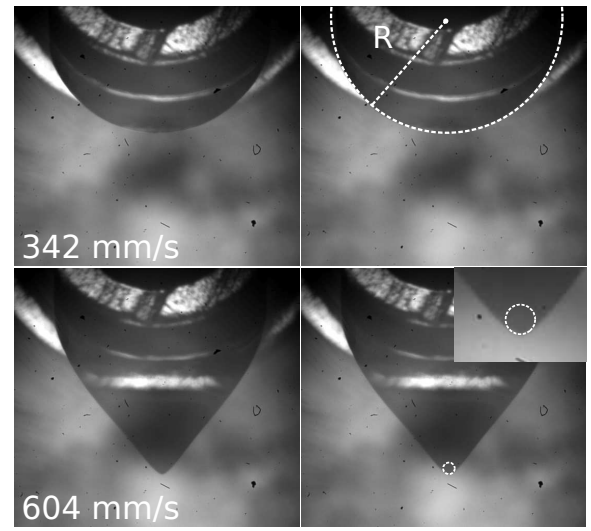

b)

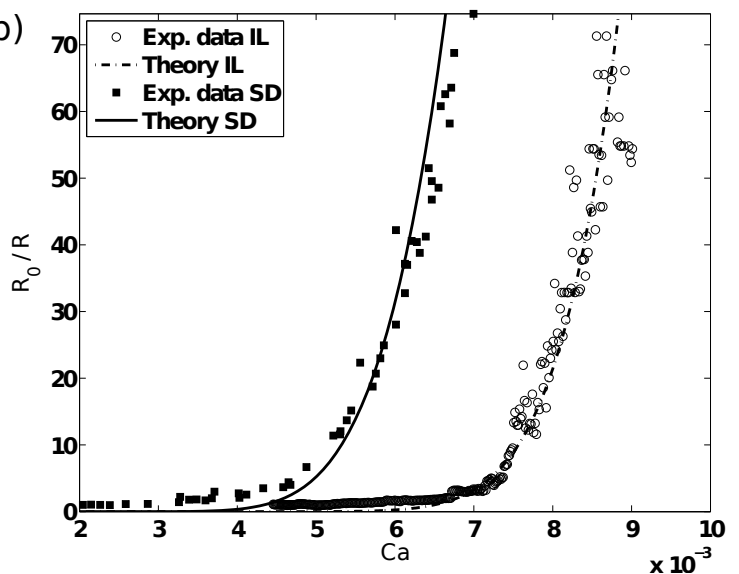

c)

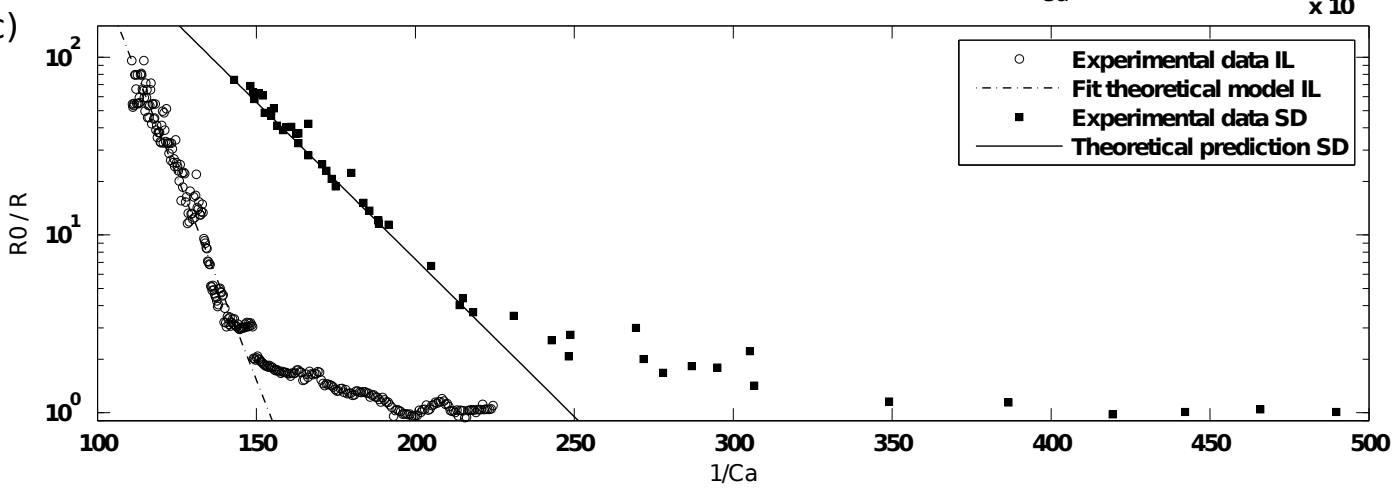

Fig. 5: Measurement of the tip curvature. a) images of typical circle fits to the receding contact line, including the definition of the radius of curvature $\mathrm{R}$. b) tip curvature $R_{0} / R$ versus Ca. Data are normalized by the initial radius of curvature $R_{0}$. Circles and squares denote data from Immersion Lithography (IL) and drops sliding down an incline (SD), respectively. c) Same as in b) plotted on a logarithmic scale to reveal the exponential behaviour (6). Solid and dashed lines are the fits with $\ell \sim 0.1 \mathrm{~nm}$ with $A=0.09$ and $\ell \sim 9 \mathrm{~nm}$ with $A=0.05$ for the Immersion Lithography and sliding droplets, respectively.

The results are shown in figure 5b, where we plot the curvature $R_{0} / R$ as a function of contact line speed Ca. Data are normalized by the initial radius, $R_{0}=0.75 \mathrm{~mm}$ for Immersion Lithography, and $R_{0}=1.63 \mathrm{~mm}$ for sliding drops. We find that the curvature remains nearly constant at low speeds, but observe a dramatic increase when the corner is formed. The curvature increases by almost 2 decades over a small range in Ca. The smallest measured tip size before instabilities, is of the order of $10 \mu \mathrm{m}$ and $20 \mu \mathrm{m}$, for Immersion Lithography data and sliding droplets, respectively. 
To test the theoretical prediction (5), we fitted the data with

$$
R=\ell \exp \left(\frac{A}{\mathrm{Ca}}\right) .
$$

These are plotted as solid and dashed lines in figure 5b and 5c, and provide a good description of the experimental data. The exponential behaviour is best revealed on a semi-logarithmic plot (figure 5c), where the slope of the linear fit represents the exponential prefactor $A$ and the offset the characteristic microscopic length scale. The former is found to be of the order nano meters/Ångström (as expected for the microscopic length scale) for both experimental data sets. The fitted values for Immersion Lithography data are $\ell \sim 0.1 \mathrm{~nm}$ and $A \approx 0.09$, while for sliding droplets, $\ell \sim 10 \mathrm{~nm}$ and $A \approx 0.05$. Note that these values are very sensitive to the details of the fit. According to the model (5), one should find $A \approx \theta_{0}^{3} / 9$. The data are indeed consistent with the static contact angle $\theta_{0}=45^{\circ}$ for the viscous sliding drops. In case of the Immersion Lithography data, however, the values of $A$ corresponds to $\theta_{0}=56^{\circ}$, which is not in accordance with the static receding contact angle $\left(\theta_{0}=65^{\circ}\right)$. We come back to this issue: the difference between water and silicon oil, in the Discussion.

\subsection{Corner opening angle}

The final characteristic of the corner is the top view angle $\phi$. Experimentally, however, there is a limited range of $\mathrm{Ca}$ where $\phi$ is a well defined quantity. This is clearly visible from the images in figure 1d). The shape of the contact line is rounded at low capillary numbers (left image). At intermediate velocities (center image), the contact line becomes distinctly "v"-shaped, resulting in two straight contact lines with a well defined angle $\phi$. At higher velocities (right image) both straight contact lines become inflected, meaning that the opening angle $\phi$ will depend on the position of measurement. Finally, the tail gives way to a rivulet like structure ultimately becoming unstable. In order to be consistent with the measurement of $\theta$, we measure $\phi$ at an equal distance from the tip where also $\theta$ was measured in the side view. The angle is measured from the intersecting tangent lines to both sides of the v-shaped contact line. The tangent lines are obtained by a similar method as used in determination of the dynamic contact angle.

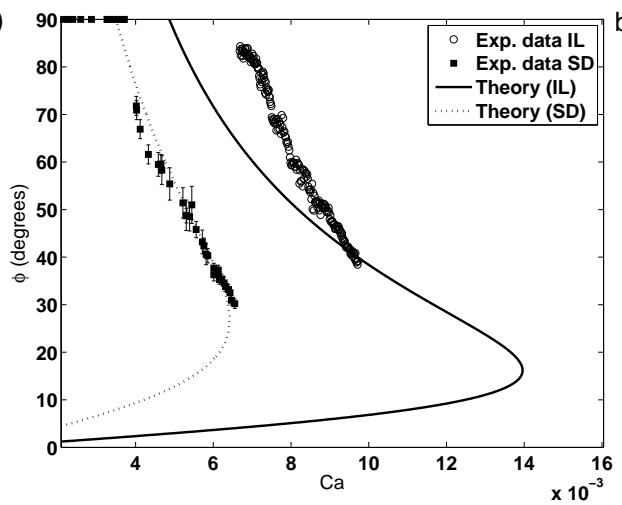

b)

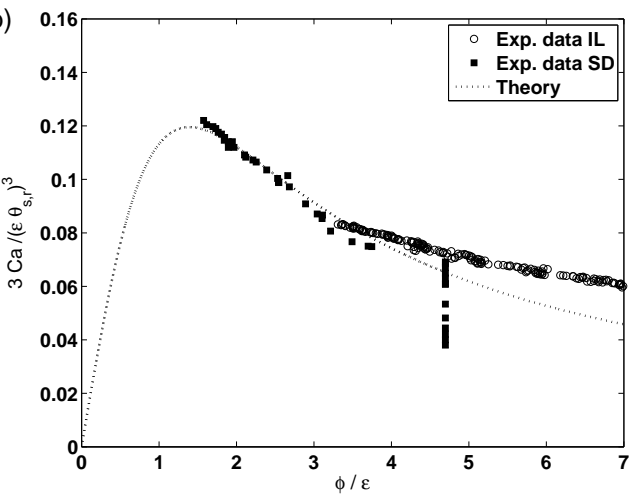

Fig. 6: The measured opening angle $\phi$ as a function of the capillary number Ca. The circles and squares shown represent the measured value of $\phi$ for the Immersion Lithography setup (IL) and the sliding droplet experiments (SD). While the lines are the predictions based on theory (3). a) measured angle $\phi$ versus Ca on a normal linear scale. b) Normalized Ca versus the normalized $\phi$. Ca rescaled by $\theta_{0}$ and $\epsilon(\epsilon=1 / \sqrt{\ln (x / \ell)})$ versus $\phi$ rescaled by $\epsilon$.

Figures $6 \mathrm{a}$ shows the measured values of $\phi$ of both experimental data sets. Clearly, the angle $\phi$ decreases with increasing capillary number. The shift in $\mathrm{Ca}$ is once more related to the 
difference in surface wettability for the Immersion Lithography and sliding drop experiments. As suggested by [17], however, the data can be rescaled by using the lubrication prediction (3). This is shown in figure $6 \mathrm{~b}$, giving a reasonable collapse of the two sets of data. We remark, however, that the theory significantly overestimates the critical speed for drop deposition.

Our simultaneous measurement of $\phi$ and $\theta$ also allows for an experimental verification of the remarkable relation between both angles as given by (2). In figure 7, the experimental data of the immersion lithography is plotted as circles together with the approximate analytical solution (2) and the exact numerical solution, represented as dashed and solid lines respectively. The trend is very similar and is almost directly comparable to the data from sliding droplet experiments done by Peters et al. [20]. However, it should be emphasized that these authors measure $\phi$ at a more distant position from the tip, which might be a cause for the small shift $[14,16]$.

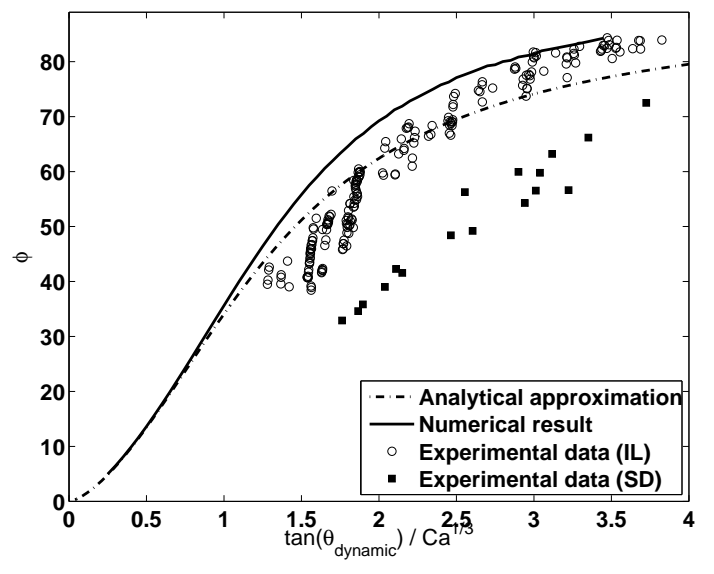

Fig. 7: Opening angle $\phi$ versus $\tan (\theta) / \mathrm{Ca}^{1 / 3}$, to compare the experimental data with the approximate prediction (7), shown as a dashed line. The solid line is the full numerical solution of the self-similar corner model [15]. Experimental data are shown as circles and squares from the Immersion Lithography setup (IL) and sliding droplet setup (SD), respectively.

\section{Discussion}

In this paper we compared v-shaped receding contact lines appearing in Immersion Lithography to the tails of drops sliding down an incline. We monitored the interface shapes for increasing contact line speed, using side view and bottom view imaging. We found that the receding contact lines in both systems behave very similarly, despite the difference in liquids (water versus silicon oil) and the difference in contact line driving. In particular, we find that the contact angle departs from the Cox-Voinov behaviour when the corner shape sets in, which can be attributed to a change from a two-dimensional to a fully three-dimensional interface. This transition is well described by a corner model based on the lubrication approximation, previously proposed for sliding drops.

There is, however, an interesting quantitative difference between water and silicon oil. For water, the logarithmic factor of the Cox-Voinov relation was found to be unphysically large, $\ln (x / \ell) \approx 23$. Namely, this would imply a microscopic length $\ell \approx 2.6 \cdot 10^{-15} \mathrm{~m}$ at which the viscous singularity is regularized. Similar values were found for sliding water drops [13], so we believe it to be due to the liquid rather than to the experimental configuration. A plausible explanation for this discrepancy lies in the relatively low viscosity of water - typical Reynolds numbers based on the drop size are of order $\mathcal{O}(100)$. By contrast, inertial effects are not important for the more viscous silicon oil drops, nor are they taken into account in 
the lubrication description. Despite this, our measurements of corner tip curvature do provide strong support for a 'viscous' logarithmic dissipation factor, also in the case of water: the exponential increase of curvature arises from inversion of the logarithm, and is indeed confirmed experimentally. This intriguing issue deserves to be explored in future work.

\section{Acknowledgements}

This work is part of the research programme of the Foundation for Fundamental Research on Matter (FOM), which is financially supported by the Netherlands Organisation for Scientific Research (NWO).

\section{References}

1. D. Bonn, J. Eggers, J. Indekeu, J. Meunier, E. Rolley, Rev. Mod. Phys. 81, (2009) pp. 739-805

2. Berg, J.C. Wettability (Marcel Dekker INC, New York, 1993) pg 318-319

3. Blake, T.D., Dobson, R.A. and Ruschak, K.J., Journal of Colloid and Interface Science 279, (2004) pp. 198-205

4. Voinov, O.V., Fluid Dyn. 11, (1976) pp. 714-721

5. Cox, R.G., J.Fluid Mech. 168, (1986) pp. 169-194

6. Derjaguin, B.V. and Levi, S.M., Film coating theory (Focal Press, London 1964)

7. Eggers, J., Physical Review Letters 93, (2004) 094502

8. Landau, L.D. and Levich, B.V., Acta Physicochimica URSS 17, (1942) 42.

9. Golestanian, R. and Raphael E., Europhys. Lett. 55, (2001) pp. 228234

10. Snoeijer, J.H., Delon, G., Fermigier, M. and Andreotti, B., Physical Review Letters 96, (2006) 174504

11. Snoeijer, J.H., Andreotti, B., Delon, G. and Fermigier, M., Journal of Fluid Mechanics 579, (2007) pp. 6383

12. Blake, T.D. and Ruschak, K.J., Nature 282, (1979) pp. 489-491

13. Podgorski, T., Flesselles, J.-M. and Limat, L., Physical Review Letters 87, (2001) 036102

14. Le Grand, N., Daerr, A. and Limat L., J. Fluid Mech. 541, (2005) pp.293-315

15. Limat, L. and Stone, H.A., Europhysics Letters 65 (3), (2004) pp.365-371

16. Snoeijer, J.H., Rio, E., Le Grand, N. and Limat, L., Physics of Fluids 17, (2005) 072101

17. Snoeijer, J.H., Le Grand, N., Limat, L., Stone, H.A. and Eggers, J., Physics of Fluids 19, (2007) 042104

18. Burnett, J.H., Kaplana, S.G., Shirleya, E.L., Tompkins, P.J. and Webb, J.E., Proceedings of SPIE 5754 Optical Microlithography XVIII

19. Riepen, M., Evangelista, F., and Donders, S., Proceedings of the 1st European Conference on Microfluidics, (2008)

20. Peters, I. , Snoeijer, J.H., Daerr, A. and Limat, L., Physical Review Letters 103, (2009) 114501

21. Huh, C. and Scriven, L.E., Journal of Colloid and Interface Science 35, (1971) pp. 85-101

22. Decker, E. L., Frank, B., Suo, Y. and Garoff, S., Colloids and Surfaces 156, (1999) pp. 177-189

23. Dussan V., E. B., Rame', E. and Garoff, S., J. Fluid Mech. 230, (1991) pp. 97-116

24. Marsh, J.A., S. Garoff and Dussan V., E. B., Physical Review Letters 70, (1993) pp. 2778-2781

25. Canny, J., IEEE TRANSACTIONS ON PATTERN ANALYSIS AND MACHINE INTELLIGENCE PAMI-8, (1986) pp. 679-698

26. Maleki, M., Reyssat, E., Quéré, D. and Golestanian, R., Langmui 23, (2007) pp. 10116-10122 27. N. Le Grand-Piteira, Ruissellement avec effets de mouillage: GOUTTES ET MANDRES SUR UN PLAN INCLIN (Thesis, Paris 2006) 